\title{
Effects of preoperative physiotherapy in hip osteoarthritis patients awaiting total hip replacement
}

\author{
Anna Czyżewska, Wojciech M. Glinkowski, Katarzyna Walesiak, Karolina Krawczak, \\ Dominika Cabaj, Andrzej Górecki
}

Chair and Department of Orthopaedics and Traumatology of Locomotor System, Centre of Excellence "TeleOrto", Medical University of Warsaw, Warsaw, Poland

Submitted: 14 February 2012

Accepted: 11 June 2012

Arch Med Sci 2014; 10, 5: 985-991

DOI: 10.5114/aoms.2014.46218

Copyright @ 2014 Termedia \& Banach

\begin{abstract}
Introduction: The World Health Organization (WHO) claimed osteoarthritis as a civilization-related disease. The effectiveness of preoperative physiotherapy among patients suffering hip osteoarthritis (OA) at the end of their conservative treatment is rarely described in the literature. The aim of this study was to assess the quality of life and musculoskeletal health status of patients who received preoperative physiotherapy before total hip replacement (THR) surgery within a year prior to admission for a scheduled THR and those who did not.

Material and methods: Forty-five patients, admitted to the Department of Orthopaedics and Traumatology of Locomotor System for elective total hip replacement surgery, were recruited for this study. The assessment consisted of a detailed interview using various questionnaires: the Harris Hip Score (HHS), the Western Ontario and McMaster Universities Osteoarthritis Index (WOMAC), the 36-Item Short Form Health Survey (SF-36), and the Hip disability and Osteoarthritis Outcome Score (HOOS), as well as physical examination. Patients were assigned to groups based on their attendance of preoperative physiotherapy within a year prior to surgery.

Results: Among patients who received preoperative physiotherapy a significant improvement was found for pain, daily functioning, vitality, psychological health, social life, and (active and passive) internal rotation $(p<0.05)$. Conclusions: Patients are not routinely referred to physiotherapy within a year before total hip replacement surgery. This study confirmed that pre-operative physiotherapy may have a positive influence on selected musculoskeletal system status indicators and quality of life in hip osteoarthritis patients awaiting surgery.
\end{abstract}

Key words: preoperative physiotherapy, rehabilitation, hip, osteoarthritis, coxarthrosis.

\section{Introduction}

Osteoarthritis (OA) remains one of the most debilitating musculoskeletal disorders among the elderly population [1, 2]. The OA produces a variety of serious social, health and economic problems [2]. The World Health Organization (WHO) claims that OA is a civilization-related disease [3]. The prevalence of symptomatic OA is about $3 \%$ of the elderly population worldwide [4]. Murphy et al. [5] estimated the lifetime risk of symptomatic hip osteoarthritis (OA). They found $25.3 \%$ lifetime risk of

\author{
Corresponding author: \\ Wojciech M. Glinkowski MD, PhD \\ Chair and Department \\ of Orthopaedics \\ and Traumatology \\ of Locomotor System \\ Center of Excellence "TeleOrto" \\ Medical University of Warsaw \\ 4 Lindleya St \\ 02-005 Warsaw, Poland \\ Phone: +48 601230 577, \\ +4822502 1197 \\ Fax: +48 225022100 \\ E-mail: w.glinkowski@gmail.com
}


symptomatic hip OA regardless of sex, race, highest educational attainment, and hip injury history.

It is estimated that OA occurs in $10 \%$ of males and $18 \%$ of females [6]. This degenerative disease occurs more frequently in hip and knee joints [6]. In the population over 75 years old approximately $10 \%$ suffers from hip OA [7]. Coxarthrosis usually presents in women over 50 years old $[1,2]$. Delayed diagnosis of disease, and lack of treatment in its early stages, may lead to its rapid progress, which often delays recovery $[2,6,8]$. Patients complain about pain, a limited range of joint motion, decreased muscle strength, limited functional daily living, and decreased quality of life [9]. Patients at the end stage of conservative treatment suffer significant pain and disability. Degenerative characteristics of the disease and pain force the physician and patient to consider surgery $[1,10$, 11]. The population of patients having undergone total hip replacement is large, rising yearly [12].

Physiotherapy is recommended for management of OA $[2,13,14]$, in the preoperative period, as one of the treatment options [14, 15]. Twelve modalities are recommended by Osteoarthritis Research Society International (OARSI): education and self-management, regular telephone contact, referral to a physical therapist, aerobic, muscle strengthening and water-based exercises, weight reduction, walking aids, knee braces, footwear and insoles, thermal modalities, transcutaneous electrical nerve stimulation, and acupuncture [16]. Preoperative rehabilitation may significantly significantly enhance clinical status of patients suffering pulmonary diseases [17].

The effectiveness of preoperative physiotherapy among patients suffering from $\mathrm{OA}$ is not frequently described in the literature $[6,8,14,18-21]$.

The aim of the study was to assess the health status of patients in several domains of quality of life and physical measurements at the end of their conservative treatment, before total hip replacement surgery, depending on participation versus nonparticipation in preoperative physiotherapy.

\section{Material and methods}

Forty-five patients, admitted to the Chair and Department of Orthopaedics and Traumatology of Locomotor System for total hip replacement (THR) surgery, were recruited to this study from January to May 2010. The study was approved by the Bioethical Committee and carried out in accordance with the International Ethical Guidelines and Declaration of Helsinki. Patients' data were analyzed according to their history of receiving preoperative physiotherapy and divided into two groups: preoperative physiotherapy "receivers" and "non-receivers". The disability and quality of life were assessed by general health and musculoskeletal status oriented questionnaires: Harris Hip Score (HHS) [22], Western Ontario and McMaster Universities Osteoarthritis Index (WOMAC) [23], Short Form 36 (SF-36) [24, 25], Hip disability and Osteoarthritis Outcome Score (HOOS) [26] and VAS (Visual Analogue Pain Scale). Patients were surveyed about their participation in preoperative physiotherapy, its duration, individual effectiveness and the willingness to continue physiotherapy in the postoperative period and the type of preoperative treatment. Their radiographs presented the hip OA severity 3 and 4 grade in Kellgren and Lawrence's scale. Patients were assigned to the preoperative physiotherapy "receivers" group if the physiotherapy programme duration was 35 days or longer.

The physical examination included the assessment of joint range of motion measured, the muscle strength, and lower extremity measurements (circumferences and lengths). Range of motion (ROM) of the hip was measured in degrees and muscle strength was scored accordingly to Lovett's scale. Circumferences were measured as follows: Gluteal 1 (P1) from the pubic symphysis to the greater trochanter, Gluteal 2 (P2) from the pubic symphysis to the intergluteal furrow. Femoral circumference 1 (U1) and 2 (U2) were measured at the largest circumference level of the thigh and at the vastus medialis obliquus level, respectively. Circumferences were measured in centimetres. The range of passive and active motion (ROM) and muscular strength were measured in flexion, extension, abduction, adduction, internal and external rotation. Differences in measured values were calculated for affected versus contralateral extremity. The clinical assessment was blindly performed regardless of the study group. All results were recorded in an Excel spreadsheet.

\section{Statistical analysis}

Statistical analysis was performed using Statistica 9.0 (StatSoft) software. The distribution of variables was assessed using the Shapiro-Wilk test. The significance of differences was tested with Student's $t$-test and the non-parametric Mann-Whitney U-test.

\section{Results}

In the group of 45 patients (32 females, and 13 males), 27 received preoperative physiotherapy whereas 18 did not. The average age of patients was $59 \pm 10.79$ years). Females were at the average age of 58 years and males 64 years. The average waiting time before admission for the surgery was 14 months. Among physiotherapy "receivers" within one year before admission 24 (89\%) received therapeutic exercises and 13 (48\%) received physical therapy. Ten patients (37\%) received therapeutic exercises combined with physi- 
cal therapy. Sixteen patients received preoperative physiotherapy at their own request and initiative (59\%), and others (41\%) were referred by a physician. Preoperative physiotherapies were given in various health care facilities as shown in Figure 1.

Three patients (11\%) noticed a significant improvement in health status. Fourteen patients (52\%) achieved little improvement after preoperative physiotherapy. Nine patients (32\%) did not observe any difference in their overall health status and only 1 patient (4\%) observed a slight deterioration. The average values and their standard deviations of scores from questionnaires obtained by patients are presented in Table I. The average scores were significantly better for the study group than the control group (except for the VAS scale).

Average values and standard deviations of the physical examination results for the study and control groups are shown in Table II. Significant differences of studied variables are shown in Table III.

Patients reported their current health status deterioration regardless of physiotherapy participation within 1 year prior to hospitalization in the SF-36 questionnaire.

Moreover, from among the "non-receivers", 15 (83.33\%) patients expressed their interest in pre-

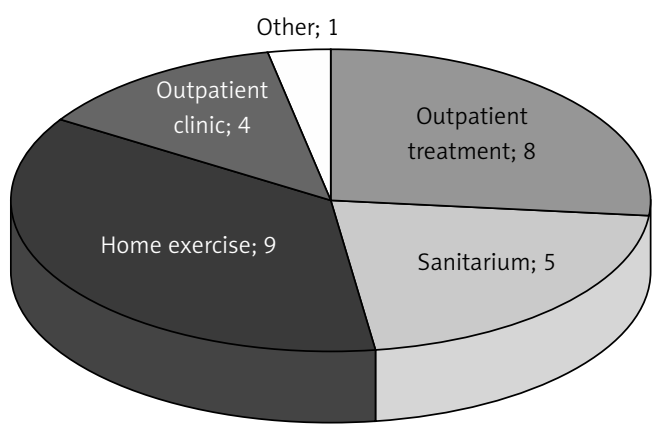

Figure 1. Facilities where patients received their preoperative physiotherapy

operative physiotherapy if given the opportunity. All patients expressed their interest in postoperative physiotherapy. Twenty patients (44.44\%) presented their willingness to exercise at home under remote supervision and monitoring by a physiotherapist over the Internet (telerehabilitation).

\section{Discussion}

The current study shows significant differences of selected variables representing health status and musculoskeletal condition gained after physiotherapy within 1 year of waiting for THR surgery.

Table I. Characteristics of scores obtained for the study group and the control group

\begin{tabular}{|lcc|}
\hline Variable & Investigated group $(n=27)$ & Control group $(n=18)$ \\
\hline HHS & $42.26 \pm 11.77$ & $37.06 \pm 11.24$ \\
\hline HOOS symptoms/stiffness & $41.67 \pm 15.44$ & $35.28 \pm 17.45$ \\
\hline HOOS pain & $41.02 \pm 17.25$ & $28.33 \pm 17.34$ \\
\hline HOOS activity daily living & $39.98 \pm 18.82$ & $26.47 \pm 19.13$ \\
\hline HOOS sport and recreation & $15.51 \pm 14.44$ & $11.46 \pm 16.22$ \\
\hline HOOS quality of life & $21.3 \pm 13.9$ & $15.63 \pm 14.74$ \\
\hline WOMAC pain & $46.67 \pm 17.97$ & $37.5 \pm 22.57$ \\
\hline WOMAC stiffness & $45.37 \pm 19.66$ & $40.97 \pm 27.39$ \\
\hline WOMAC function & $37.15 \pm 15.71$ & $28.19 \pm 17.98$ \\
\hline SF-36 physical functioning & $25.93 \pm 20.48$ & $20.83 \pm 16.83$ \\
\hline SF-36 role limitations due to physical problems & $24.07 \pm 37$ & $13.89 \pm 26.04$ \\
\hline SF-36 role limitations due to emotional problems & $37.04 \pm 44.66$ & $16.67 \pm 30.78$ \\
\hline SF-36 vitality & $46.48 \pm 12.47$ & $35.56 \pm 18.86$ \\
\hline SF-36 mental health & $60.59 \pm 17.64$ & $46.89 \pm 21.11$ \\
\hline SF-36 social functioning & $48.15 \pm 25.64$ & $31.94 \pm 25.80$ \\
\hline SF-36 bodily pain & $30.28 \pm 20.75$ & $21.81 \pm 15.07$ \\
\hline SF-36 general health perceptions & $50.19 \pm 15.72$ & $44.72 \pm 17.10$ \\
\hline SF-36 current state of health/state of health & $23.15 \pm 19.52$ & $13.89 \pm 19.60$ \\
\hline year prior surgery & $5.11 \pm 1.55$ & 5.28 \\
\hline VAS & & \\
\hline
\end{tabular}


Table II. Average values and standard deviations of the physical examination results for the study and control groups

\begin{tabular}{|c|c|c|}
\hline Variable & Investigated group $(n=27)$ & Control group $(n=18)$ \\
\hline Difference: P1 & $-0.04 \pm 2.08$ & $-0.17 \pm 1.82$ \\
\hline Difference: P2 & $0.44 \pm 1.69$ & $0.78 \pm 1.44$ \\
\hline Difference: U1 & $2.44 \pm 3.85$ & $0.89 \pm 2.91$ \\
\hline Difference: U2 & $0.85 \pm 3.45$ & $-0.56 \pm 4.42$ \\
\hline Difference: direct leg - length & $2 \pm 3.21$ & $-0.67 \pm 2.61$ \\
\hline Difference: indirect leg - length & $1.44 \pm 2.67$ & $-0.06 \pm 2.41$ \\
\hline Difference: active flexion rotation rom & $25.19 \pm 26.69$ & $16.39 \pm 30.19$ \\
\hline Difference: passive flexion rotation rom & $19.81 \pm 20.96$ & $19.72 \pm 33.54$ \\
\hline Difference: active extention rotation rom & $5.74 \pm 6.75$ & $3.06 \pm 5.98$ \\
\hline Difference: passive extention rotation rom & $6.3 \pm 8.16$ & $3.06 \pm 7.7$ \\
\hline Difference: active abduction rotation rom & $8.52 \pm 7.18$ & $8.06 \pm 10.02$ \\
\hline Difference: passive abduction rotation rom & $8.15 \pm 7.61$ & $10.56 \pm 10.13$ \\
\hline Difference: active adduction rotation rom & $4.07 \pm 4.61$ & $4.17 \pm 8.27$ \\
\hline Difference: passive adduction rotation rom & $4.07 \pm 5.89$ & $5 \pm 7.07$ \\
\hline Difference: active int. rotation rom & $11.48 \pm 10.08$ & $4.17 \pm 9.12$ \\
\hline Difference: passive int. rotation rom & $12.22 \pm 10.41$ & $3.61 \pm 9.36$ \\
\hline Difference: active ext. rotation rom & $5.56 \pm 9.34$ & $10.56 \pm 7.84$ \\
\hline Difference: passive ext. rotation rom & $6.49 \pm 9.49$ & $8.89 \pm 8.14$ \\
\hline Difference: flexion muscle strength & $0.63 \pm 0.69$ & $0.61 \pm 0.76$ \\
\hline Difference: extention muscle strength & $0.65 \pm 1.13$ & $0.03 \pm 0.74$ \\
\hline Difference: abduction muscle strength & $0.59 \pm 0.73$ & $0.83 \pm 0.86$ \\
\hline Difference: adduction muscle strength & $0.33 \pm 0.92$ & $0.58 \pm 0.81$ \\
\hline Difference: internal rotation muscle strength & $0.74 \pm 0.89$ & $0.31 \pm 0.88$ \\
\hline Difference: external rotation muscle strength & $0.33 \pm 0.72$ & $0.42 \pm 0.83$ \\
\hline
\end{tabular}

Table III. Variables found significantly different between physiotherapy "receivers" and "non-receivers" (bold represents significant difference)

\begin{tabular}{|lcccc|}
\hline Variable & $\begin{array}{c}\text { AVG } \\
\text { investigated group }\end{array}$ & $\begin{array}{c}\text { AVG } \\
\text { control group }\end{array}$ & Test $T$ & Value of $p$ \\
\hline HOOS activity daily living & 39.97810 & 26.47059 & 2.34315 & 0.023816 \\
\hline SF-36 vitality & 46.48148 & 35.55556 & 2.34457 & 0.023737 \\
\hline SF-36 mental health & 60.59259 & 46.88889 & 2.35955 & 0.022907 \\
\hline SF-36 social functioning & 48.14815 & 31.94444 & 2.07153 & 0.044343 \\
\hline Difference: functional limb - length & 2.00000 & -0.66667 & 2.93258 & 0.005370 \\
\hline Difference: active internal rotation rom & 11.48148 & 4.16667 & 2.47599 & 0.017301 \\
\hline Difference: passive internal rotation rom & 12.22222 & 3.61111 & 2.82762 & 0.007092 \\
\hline
\end{tabular}

The strength of this study is the focus on the group of patients directly before THR waiting a long time for the surgery. This study has some limitations. First of all the study groups were not cohorts. Pa- tients were asked to retrospect their physiotherapy participation. Another limitation comes from inequality of the physiotherapy programmes that were received by patients. The quality, protocols 
and intensity of physiotherapy given to patients were not standardized. Most often, therapeutic exercise treatment guidelines are based only on the opinion of the individual professionals [27]. The only comparable descriptor of the physiotherapy program was its duration. Regardless of the physiotherapy protocol, the results achieved in this study strongly suggest its positive influence that may make the patient more fit and strong before the extensive surgery. This study also shows that hip OA patients are not routinely referred for preoperative physiotherapy when they are on the waiting list for THR surgery. The majority of patients from both groups expressed their interest in postoperative physiotherapy.

Patients awaiting hip replacement surgery suffered significant pain, functional disability and low quality of life. They predominantly reported worsening of their current health status within the last year prior to the surgery according to the SF-36. Quality of life oriented questionnaires showed that more than a half of examined patients gained benefits from exercising or exercising and physical therapy before surgery.

Results of randomized controlled trials demonstrated slight pain relief among patients with symptomatic hip OA, who received preoperative exercises [28].

Ferrara et al. [29] observed significant differences in pain expressed in the values of the visual analogue scale of pain (VAS), physical functioning according to the SF-36 questionnaire and external rotation ROM in the hip $(p<0.05)$ in patients undergoing THR surgery who received preoperative physiotherapy. Notable improvement was also described for the hip abductor muscle strength. The considerable differences between the results obtained in WOMAC and HHS questionnaires were not confirmed in the current study.

In our study patients undergoing physiotherapy significantly improved in pain and activity/daily living subscales of the HOOS questionnaire and vitality, mental health, and social life domains of the SF-36 questionnaire. The difference in active and passive internal rotation was found remarkably better for physiotherapy "receivers".

Several papers have indicated that use of therapeutic exercises in conjunction with physical therapy among patients with hip osteoarthritis is effective and recommended for improving joint range of motion and reducing pain $[15,16,30]$. However, our observation of the pain VAS scores did not support that. It was found that preoperative physiotherapy may have a beneficial effect on patients' health status after THR [1]. Moreover, traditional rehabilitation based on range of joint motion or isometric muscle strengthening exercises with walking practice may be less effective than more functional rehabilitation [31]. Patients participating in preoperative rehabilitation have less fear of the surgery, a shorter period of convalescence and faster recovery of independence in locomotion and self-care [29], which should be considered as one of the main arguments for implementation of rehabilitation in the period of waiting for surgery $[1,32,33]$.

Some authors contend that a preoperative exercise programme can improve physical condition, patient muscle strength before surgery and other quality of life parameters (for example those included in the WOMAC questionnaire) [8]. In the current study similar improvements were confirmed for selected functional parameters. A few authors did not observe beneficial effects of preoperative physiotherapy and education on the patients' health after surgery $[8,19,29]$. Preoperative physiotherapy may have an effect on shortening hospital stay $[8,29]$. On the basis of our study it is difficult to question the presented opinion.

Hopman-Rock and Westhoff [34] found significant time effects of evaluated self-management programmes for patients with hip OA for pain, quality of life, strength of the left quadriceps muscle, knowledge, self-efficacy, body mass index, physically active lifestyle, and visits to the physical therapist. Their programme included 6 weeks of rehabilitation that consisted of health education and physical exercises. On the other hand, the study of Pisters et al. [35] did not confirm the long term ( $\geq 6$ months after surgery) positive results of exercises on pain and physical function in patients with coxarthrosis. Tak et al. [36] applied an 8-week strength exercise training programme (using fitness equipment) of $1 \mathrm{~h}$ per week, a home exercise programme and individualized occupational therapy consultation with dietary advice. The study showed a significant improvement in hip function in Harris Hip Score in the investigated group at the post-test $(p<0.05)$. Nevertheless, the value of improved results dropped back toward baseline at the follow-up assessment.

Home exercises improve the quality of life and reduce disability of patients with hip osteoarthritis [10]. Our own research shows that patients undergoing preoperative physiotherapy often participated in home-based exercises (33\%). Galea et al. [37] confirmed the effectiveness of strengthening for home- and centre-based exercises. Patients after THR achieved significant improvement in quality of life, stair climbing, walking speed increase, cadence, step length and its symmetry in both groups.

The application of modern information technology in medicine is increasing the presence of telemedicine services in health care, including 
telerehabilitation. It delivers added value for rehabilitation services. Telerehabilitation can become a kind of additional treatment for patients who perform home-based exercises [38, 39]. Our patients were considerably interested in a remotely supervised rehabilitation programme for patients with hip osteoarthritis (44\%).

We assume that our approach presented in this study is relatively rare. The effectiveness of preoperative physiotherapy in end-stage conservative treatment of coxarthrosis is not often described in the literature. The protocol of this study covered a wide range of assessments consisting of several questionnaires concerning physical and mental health and quality of life. The majority of them supplemented each other rather than overlapped. Several issues touched in this study require further investigation because of the rather preliminary character of this study.

Due to the integrated, collaborative and personalized approach to the treatment of locomotor system diseases (orthopaedic and rehabilitation), the efficacy of preoperative physiotherapy should be a subject of further research.

In conclusion, routine referral for physiotherapy was found unusual for patients suffering hip joint arthrosis (only $60 \%$ of examined patients). The majority of patients reported significant deterioration in their health status within 1 year before admission for THR surgery. Preoperative physiotherapy may have a positive influence on selected musculoskeletal system status indicators and the quality of life. During the period of waiting for surgery the home-based therapeutic exercises are the most common way to participate in rehabilitation. Hip osteoarthritis patients are often interested in participation in preoperative $(83 \%)$ and always in postoperative physiotherapy (100\%). Patients also express their interest in telerehabilitation (44\%).

\section{Acknowledgments}

This study was partially supported by the project CLEAR ((ICT-PSP-224985) Clinical Leading Environment for the Assessment and validation of Rehabilitation Protocols for home care).

\section{References}

1. Minns Lowe CJ, Barker KL, Dewey ME, Sackley CM. Effectiveness of physiotherapy exercise following hip arthroplasty for osteoarthritis: a systematic review of clinical trials. BMC Musculoskelet Disord 2009; 10: 98.

2. DeLisa J, Gans B, Walsh N, et al. Physical medicine and rehabilitation: principles and practice. 4th ed. 2005; 4.

3. Woolf AD, Pfleger B. Burden of major musculoskeletal conditions. Bulletin of the World Health Organization 2003; 81: 646-56.
4. Felson DT. An update on the pathogenesis and epidemiology of osteoarthritis. Radiol Clin North Am 2004 42: $1-9$

5. Murphy LB, Helmick CG, Schwartz TA, et al. One in four people may develop symptomatic hip osteoarthritis in his or her lifetime. Osteoarthritis Cartilage 2010; 18: 1372-9.

6. Escalante Y, Saavedra JM, García-Hermoso A, Silva AJ, Barbosa TM. Physical exercise and reduction of pain in adults with lower limb osteoarthritis: a systematic review. J Back Musculoskelet Rehabil 2010; 23: 175-86.

7. Escalante Y, Garcia-Hermoso A, Saavedra JM. Effects of exercise on functional aerobic capacity in lower limb osteoarthritis: a systematic review. J Sci Med Sport 2011; 14: $190-8$.

8. Rooks DS, Huang J, Bierbaum BE, et al. Effect of preoperative exercise on measures of functional status in men and women undergoing total hip and knee arthroplasty. Arthritis Rheum 2006; 55: 700-8.

9. Hoeksma HL, Dekker J, Ronday HK, et al. Comparison of manual therapy and exercise therapy in osteoarthritis of the hip: a randomized clinical trial. Arthritis Rheum 2004; 51: 722-9.

10. Khan F, Ng L, Gonzalez S, Hale T, Turner-Stokes L. Multidisciplinary rehabilitation programmes following joint replacement at the hip and knee in chronic arthropathy. Cochrane Database Syst Rev 2008; 2: CD004957.

11. Räsänen P, Paavolainen $P$, Sintonen $H$, et al. Effectiveness of hip or knee replacement surgery in terms of quality-adjusted life years and costs. Acta Orthop 2007; 78: 108-15

12. Dieppe P, Judge A, Williams S, et al.; EUROHIP Study Group. Variations in the pre-operative status of patients coming to primary hip replacement for osteoarthritis in European orthopaedic centres. BMC Musculoskelet Disord 2009; 10: 19.

13. Lenssen AF, de Bie RA. Role of physiotherapy in peri-operative management in total knee and hip surgery. Injury 2006; 37 Suppl. 5: S41-3.

14. Dauty M, Genty M, Ribinik P. Physical training in rehabilitation programs before and after total hip and knee arthroplasty. Ann Readapt Med Phys 2007; 50: 462-8, 455-61.

15. Hunter DJ, Felson DT. Osteoarthritis. BMJ 2006; 332: 639-42.

16. Zhang W, Moskowitz RW, Nuki G, et al. OARSI recommendations for the management of hip and knee osteoarthritis. Part II: OARSI evidence-based, expert consensus guidelines. Osteoarthritis Cartilage 2008; 16 : 137-62

17. Mujovic N, Mujovic N, Subotic D, et al. Preoperative pulmonary rehabilitation in patients with non-small cell lung cancer and chronic obstructive pulmonary disease. Arch Med Sci 2014; 10: 68-75.

18. Barbay K. Research evidence for the use of preoperative exercise in patients preparing for total hip or total knee arthroplasty. Orthop Nurs 2009; 28: 127-33.

19. Coudeyre E, Jardin C, Givron P, Ribinik P, Revel M, Rannou F. Could preoperative rehabilitation modify postoperative outcomes after total hip and knee arthroplasty? Elaboration of French clinical practice guidelines. Ann Readapt Med Phys 2007; 50: 189-97.

20. Gocen Z, Sen A, Unver B, Karatosun V, Gunal I. The effect of preoperative physiotherapy and education on the outcome of total hip replacement: a prospective randomized controlled trial. Clin Rehabil 2004; 18: 353-8. 
21. Murphy SL, Lyden AK, Smith DM, Dong Q, Koliba JF. Effects of a tailored activity pacing intervention on pain and fatigue for adults with osteoarthritis. Am J Occup Ther 2010; 64: 869-76.

22. Kirmit L, Karatosun V, Unver B, Bakirhan S, Sen A, Gocen $Z$. The reliability of hip scoring systems for total hip arthroplasty candidates: assessment by physical therapists. Clin Rehabil 2005; 19: 659-61.

23. Williams JI, Llewellyn Thomas H, Arshinoff R, Young N, Naylor CD. The burden of waiting for hip and knee replacements in Ontario. Ontario Hip and Knee Replacement Project Team. J Eval Clin Pract 1997; 3: 59-68.

24. Boardman DL, Dorey F, Thomas BJ, Lieberman JR. The accuracy of assessing total hip arthroplasty outcomes: a prospective correlation study of walking ability and 2 validated measurement devices. J Arthroplasty 2000; 15: 200-4.

25. Soderman P, Malchau $H$. Validity and reliability of Swed ish WOMAC osteoarthritis index: a self-administered disease-specific questionnaire (WOMAC) versus generic instruments (SF-36 and NHP). Acta Orthop Scand 2000; 71: 39-46.

26. Nilsdotter AK, Lohmander LS, Klässbo M, Roos EM. Hip disability and osteoarthritis outcome score (HOOS)-validity and responsiveness in total hip replacement. BMC Musculoskel Disord 2003; 4: 10.

27. Glinkowski W. Pre-surgery and post-surgery telerehabilitation for hip and knee replacement. Treatment options review and patient's attitudes towards telerehabilitation. in eChallenges e-2010 Conference Proceedings. 2010. Warsaw: IIMC International Information Management Corporation.

28. Fransen M, McConnell S, Hernandez-Molina G, Reichenbach S. Exercise for osteoarthritis of the hip. Cochrane Database Syst Rev 2009; 3: CD007912.

29. Ferrara PE, Rabini A, Maggi L, et al. Effect of pre-operative physiotherapy in patients with end-stage osteoarthritis undergoing hip arthroplasty. Clin Rehabil 2008; 22: 977-86

30. Conaghan PG, Dickson J, Grant RL. Care and management of osteoarthritis in adults: summary of NICE guidance. BMJ 2008; 336: 502-3.

31. Trudelle-Jackson E, Smith SS. Effects of a late-phase exercise program after total hip arthroplasty: a randomized controlled trial. Arch Phys Med Rehabil 2004; 85: 1056-62.

32. Peter WF, Jansen MJ, Hurkmans EJ, et al. Physiotherapy in hip and knee osteoarthritis: development of a practice guideline concerning initial assessment, treatment and evaluation. Acta Reumatol Port 2011; 36: 268-81.

33. Vukomanović A, Popović Z, Durović A, Krstić L. The effects of short-term preoperative physical therapy and education on early functional recovery of patients younger than 70 undergoing total hip arthroplasty. Vojnosanit Pregl 2008; 65: 291-7.

34. Hopman-Rock M, Westhoff MH. The effects of a health educational and exercise program for older adults with osteoarthritis for the hip or knee. J Rheumatol 2000; 27: 1947-54.

35. Pisters MF, Veenhof C, van Meeteren NL, et al. Longterm effectiveness of exercise therapy in patients with osteoarthritis of the hip or knee: a systematic review. Arthritis Rheum 2007; 57: 1245-53.

36. Tak E, Staats P, Van Hespen A, Hopman-Rock M. The effects of an exercise program for older adults with osteoarthritis of the hip. J Rheumatol 2005; 32: 1106-13.
37. Galea MP, Levinger P, Lythgo N, et al. A targeted homeand center-based exercise program for people after total hip replacement: a randomized clinical trial. Arch Phys Med Rehabil 2008; 89: 1442-7.

38. Burdea G, Popescu V, Hentz V, Colbert K. Virtual reality-based orthopedic telerehabilitation. IEEE Trans Rehabil Eng 2000; 8: 430-2.

39. Glinkowski W. Telerehabilitation - practical applications in orthopaedics and traumatology of the locomotor system. Management and Information Technologies 2008; 3: 225-41. 